\title{
Optimal control for a size-structured predator-prey model in a polluted environment
}

\author{
Tainian Zhang, ${ }^{1,}$, Zhixue Luo $^{2}$ \\ ${ }^{1}$ School of Environmental and Municipal Engineering, Lanzhou Jiaotong University, Lanzhou 730070, China \\ ${ }^{2}$ Department of Mathematics, Lanzhou Jiaotong University, Lanzhou 730070, China
}

\begin{abstract}
In this paper, we deal with an optimal harvesting problem for a periodic predator-prey hybrid system dependent on size-structure in a polluted environment. In other words, a size-dependent model in an environment with a small toxicant content has been established. The well-posedness of state system is proved by using the fixed point theorem. The necessary optimality conditions are derived by tangentnormal cone technique in nonlinear functional analysis. The existence of a unique optimal harvesting policy is verified via the Ekeland's variational principle. The optimal harvesting problem has an optimal harvesting policy, which has a Bang-Bang structure and provides a threshold for the optimal harvesting problem. Using the optimization theories and methods in mathematics to control phenomena of life. The objective function represents the total economic profit from the harvested population. Some theoretical results obtained in this paper provide a scientific theoretical basis for the practical application of the model.
\end{abstract}

\section{Introduction}

With the rapid development of the global economy, many new technologies have been applied to industrial and agricultural production activities. The invasion of toxicants is very easy to happen, and environmental pollution has become more and more serious. Bush fires in Australia, SARS, Ebola virus, AIV, H1N1 influenza, and COVID-19, etc., these phenomena are threatening the ecological balance and causing serious harm to the survival of human beings and other creatures. Therefore, it is necessary to study the effects of toxicants on biological populations. Hallam et al. proposed an idea of using dynamics methods to study ecotoxicology in the literature [1-3]. They established a toxicants-population model and studied the persistence and extinction of a population in a polluted environment. Since the 1980s, people have conducted in-depth research on the topic of ecotoxicology. Nowadays, there is a large number of literature on ecotoxicology problems [4-6], but sizestructured factors are not considered in these models.

For many populations, the individual size determines the life parameters of the individual to a large extent, such as reproductive rate, mortality, metabolic capacity and predation capacity, etc., thereby affecting the dynamic behavior of the populations. In addition, the individual size-structure can explain some phenomena that other structures cannot explain, such as the selfthinning of plants and the elastic growth of individuals. Furthermore, the individual size has good operability, such as easy measurement, convenient scientific management of ecological resources, and easy access to size-based statistical data. It is more convenient in practical applications. This kind of models has achieved remarkable results through theory, numerical calculation and experimental methods, which can be found in the literature [7-10]. Due to the influence of seasonal changes and other factors, the living environment of the populations often experience periodic changes. For the research on the optimal harvest problems dependent on individual size-structured models in a periodic environment can be referred to [11-13], in which the literature [11] discussed harvesting problem for nonlinear size-dependent population model in periodic environments. At present, only a few papers have focused on the optimal control problems of population models with size-structure and periodic effects in a polluted environment. Inspired by the above, this paper discusses the optimal harvesting problem of a periodic predator-prey system dependent on size-structure in a polluted environment.

The remaining part of this paper is as follows. The problem is described and the main methods of proof are given in the next section. The well-posedness is proved, the optimality conditions for the harvesting problem are derived, the existence of a unique optimal policy is obtained and the problem is discussed in Sect. 3. Finally, we give a short conclusion in the last section.

\section{Methodology}

We establish the following periodic predator-prey model with size-structure in a polluted environment:

\footnotetext{
* Corresponding author: tn zhang91@,163.com
} 


$$
\left\{\begin{array}{l}
\frac{\partial p_{1}(s, t)}{\partial t}+\frac{\partial\left(g_{1}(s) p_{1}(s, t)\right)}{\partial s}=f_{1}(s, t)-\mu_{1}\left(s, c_{10}(t)\right) \\
\cdot p_{1}(s, t)-\lambda_{1}(s, t) P_{2}(t) p_{1}(s, t)-u_{1}(s, t) p_{1}(s, t), \\
\frac{\partial p_{2}(s, t)}{\partial t}+\frac{\partial\left(g_{2}(s) p_{2}(s, t)\right)}{\partial s}=f_{2}(s, t)-\mu_{2}\left(s, c_{20}(t)\right) \\
\cdot p_{2}(s, t)+\lambda_{2}(s, t) P_{1}(t) p_{2}(s, t)-u_{2}(s, t) p_{2}(s, t), \\
\frac{\mathrm{d} c_{i 0}(t)}{\mathrm{d} t}=k c_{e}(t)-g c_{i 0}(t)-m c_{i 0}(t), \\
\frac{\mathrm{d} c_{e}(t)}{\mathrm{d} t}-k_{1} c_{e}\left[P_{1}(t)+P_{2}(t)\right] \\
\quad+g_{1}\left[c_{10}(t) P_{1}(t)+c_{20}(t) P_{2}(t)\right]-h c_{e}(t)+v(t), \\
g_{i}(0) p_{i}(0, t)=\int_{0}^{l} \beta_{i}\left(s, c_{i 0}(t)\right) p_{i}(s, t) \mathrm{d} s, \\
p_{i}(s, t)=p_{i}(s, t+T), \\
P_{i}(t)=\int_{0}^{l} p_{i}(s, t) \mathrm{d} s, \quad i=1,2,
\end{array}\right.
$$

where $Q=(0, l) \times R_{+}, R_{+}=(0,+\infty), l \in R_{+}$is the maximal size, $T \in R_{+}$is the habitat evolution period. Other parameters are defined as follows:

$p_{i}(s, t)$ : the density of ith population of size $s$ at time $t$.

$c_{i 0}(t)$ : the concentration of the toxicants in ith population.

$c_{e}(t)$ : the concentration of the toxicants in the environment.

The vital rates of ith populations $g_{i}(s), \mu_{i}(s, t)$ and $\beta_{i}(s, t)$ : the growth rate of the individual size, mortality and fertility, respectively.

$\lambda_{i}(s, t)$ : the interaction coefficient.

$v(t)$ : the exogenous toxicants input rate.

$P_{i}(t)$ : total number of ith population.

The $k, g, m, k_{1}, g_{i}, h$ are non-negative constants.

The $u_{i}(s, t)$ is the harvesting efforts, which belongs to

$$
\begin{gathered}
\Omega=\left\{(u, v) \in\left[L_{T}^{\infty}(Q)\right]^{2} \times L_{T}\left(R_{+}\right): 0 \leq u_{i}(s, t) \leq H,\right. \\
0 \leq v(t) \leq h, \forall(s, t) \in Q\},
\end{gathered}
$$

where $\Omega$ is admissible control set, and $\eta(s, t)=\eta(s, t+T)$, a.e. $(s, t) \in Q, \eta(t)=\eta(t+T)$, a.e. $t \in(0, T)$. In this paper, we consider the following optimal harvesting problem:

$$
\max _{(u, v) \in \Omega}\left\{J(u, v): u=\left(u_{1}(s, t), u_{2}(s, t)\right), v=v(t)\right\},
$$

where

$$
\begin{aligned}
J(u, v)= & \sum_{i=1}^{2} \int_{0}^{T} \int_{0}^{l} w_{i}(s, t) u_{i}(s, t) p_{i}(s, t) \mathrm{d} s \mathrm{~d} t \\
& -\frac{1}{2} \sum_{i=1}^{2} \int_{0}^{T} \int_{0}^{l} c_{i}\left[u_{i}(s, t)\right]^{2} \mathrm{~d} s \mathrm{~d} t-\frac{1}{2} \int_{0}^{T} c_{3}[v(t)]^{2} \mathrm{~d} t,
\end{aligned}
$$

represents the total economic profit from the harvested population. The weight function $w_{i}(s, t)$ is the selling price of an individual. The positive constant $c_{1}, c_{2}$ and $c_{3}$ are the cost factors of harvesting populations and the cost factor of curbing environmental pollution, respectively. The state $\left(p, c_{0}, c_{e}\right)$ is the solution of the system (1) corresponding to $(u, v)$.

Throughout this paper, we assume that:

$\left(A_{1}\right) g_{i} \in C^{1}[0, l), g_{i}(0)=1, \lim _{s \uparrow l} g_{i}(s)=0, g_{i}(s)>0$, and $g_{i}^{\prime}(s) \leq 0$, a. e. $s \in[0, l)$. There are constants $L_{V_{i}}>0$ such that for all $s_{1}, s_{2} \in[0, l)$ :

$$
\left|g_{i}\left(s_{1}\right)-g_{i}\left(s_{2}\right)\right| \leq L_{V_{i}}\left|x_{1}-x_{2}\right| \text {. }
$$

$\left(A_{2}\right) 0 \leq \beta_{i}\left(s, c_{i 0}(t)\right) \leq \beta^{0}\left(s, c_{i 0}(t+T)\right), 0 \leq w_{i}(s, t)=$ $w_{i}(s, t+T) \leq \bar{w}$.

$\left(A_{3}\right) \mu_{i}\left(\cdot, c_{i 0}(t)\right) \in L_{l o c}^{1}(0, l), 0 \leq \mu_{i}\left(s, c_{i 0}(t)\right)=\mu_{i}\left(s, c_{i 0}(t+T)\right)$,

$\int_{0}^{l} \mu_{i}\left(\Gamma_{i}^{-1}\left(\Gamma_{i}(l)-s\right), t-s\right) \mathrm{d} s=+\infty . \Gamma_{i}(s)=\int_{0}^{s} \frac{1}{g_{i}(\vartheta)} \mathrm{d} \vartheta$.

$\left(A_{4}\right) v(\cdot) \in L^{2}[0, T], 0 \leq v_{0} \leq v(t) \leq v_{1}<+\infty$.

$\left(A_{5}\right) g \leq k \leq g+m, v_{1} \leq h$.

$\left(A_{6}\right)\left|\beta_{i}\left(s, c_{i 0}^{1}(t)\right)-\beta_{i}\left(s, c_{i 0}^{2}(t)\right)\right| \leq L_{\beta}\left|c_{i 0}^{1}(t)-c_{i 0}^{2}(t)\right|, \mid \mu\left(s, c_{i 0}^{1}(t)\right.$

$-\mu_{i}\left(s, c_{i 0}^{2}(t)\right)\left|\leq L_{\mu}\right| c_{i 0}^{1}(t)-c_{i 0}^{2}(t) \mid, 0 \leq \lambda_{i} \leq \lambda^{0}$.

$\left(A_{7}\right) f_{i} \in L^{\infty}(Q), 0 \leq f_{i} \leq B, f_{i}(s, t)=f_{i}(s, t+T)$, for

$(s, t) \in Q$.

Next, we will prove the existence and uniqueness of non-negative solution of the toxicant-population model by the fixed point theorem, deduct the optimality conditions of the optimal harvesting problem by employing tangent-normal cone techniques, and obtain the existence of optimal harvesting policy by the Ekeland's variational principle.

\section{Experimental results and discussion}

\subsection{Well-posedness of the state system}

In this section, we discuss the well-posedness of state system (1), and first give the following definition.

Definition 3.1.1 The $\left(p_{1}(s, t), p_{2}(s, t), c_{10}(t), c_{20}(t), c_{e}(t)\right)$ is a solution of state system (1), if it is absolutely continuous along almost every characteristic curve $t-\Gamma_{i}(s)=\gamma \quad(\gamma \in \mathbf{R})[14]$ and satisfies: 


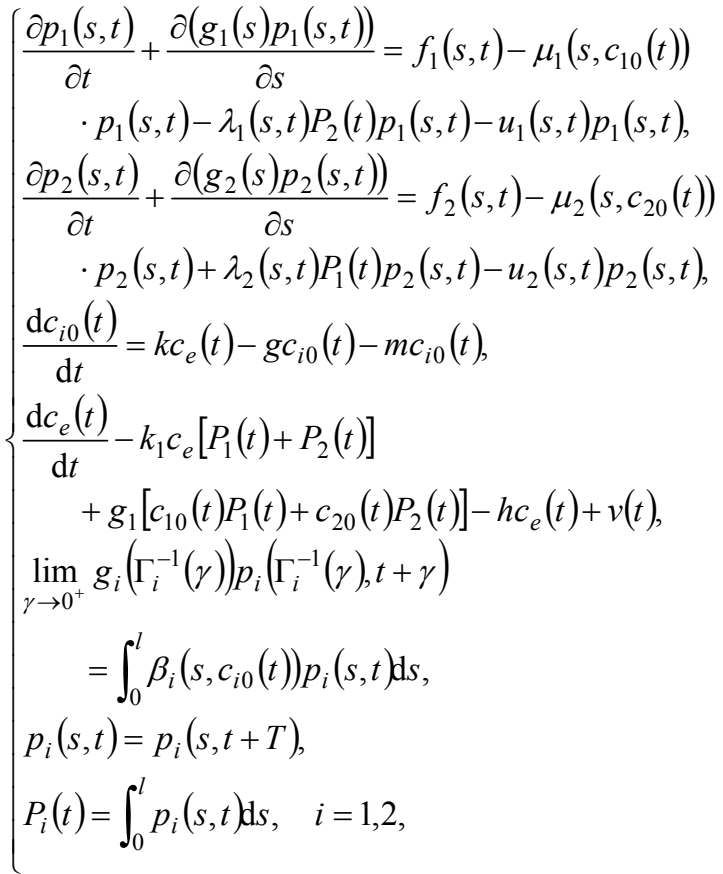

Theorem 3.1.2 Assuming $\left(A_{1}\right)-\left(A_{7}\right)$ hold, then the state system (1) has unique non-negative solution that satisfies the following conditions:

(I) $\left(p_{1}(s, t), p_{2}(s, t), c_{10}(t), c_{20}(t), c_{e}(t)\right) \in\left[L_{T}^{\infty}(Q)\right]^{2} \times\left[L^{\infty}\left(R_{+}\right)\right]^{3}$;

(II) $0 \leq c_{0}(t) \leq 1,0 \leq c_{e}(t) \leq 1, t \in R_{+}$.

Proof Without a loss of generality, suppose $u_{i}(x, t) \equiv 0$.

From definition 3.1.1, the solution of the state system (1) can be written as

$$
\begin{aligned}
& p_{i}(s, t) \\
& =b_{i}\left(t-\Gamma_{i}(s)\right) \exp \left\{-\int_{0}^{\Gamma_{i}(s)} \mu_{i}\left(\Gamma_{i}^{-1}(\tau), c_{i 0}\left(t+\tau-\Gamma_{i}(s)\right)\right)\right. \\
& +(-1)^{i+1} \lambda_{i}\left(\Gamma_{i}^{-1}(\tau), t+\tau-\Gamma_{i}(s)\right) P_{j}\left(t+\tau-\Gamma_{i}(s)\right) \\
& \left.+g_{i s}\left(\Gamma_{i}^{-1}(\tau)\right)\right\} \mathrm{d} \tau \\
& +\int_{0}^{\Gamma_{i}(s)} \exp \left\{-\int_{\tau}^{\Gamma_{i}(s)} \mu_{i}\left(\Gamma_{i}^{-1}(\sigma), c_{i 0}\left(t+\sigma-\Gamma_{i}(s)\right)\right)\right. \\
& +(-1)^{i+1} \lambda_{i}\left(\Gamma_{i}^{-1}(\sigma), t+\sigma-\Gamma_{i}(s)\right) P_{j}\left(t+\sigma-\Gamma_{i}(s)\right) \\
& \left.+g_{i s}\left(\Gamma_{i}^{-1}(\sigma)\right) \mathrm{d} \sigma\right\} f_{i}\left(\Gamma_{i}^{-1}(\tau), t+\tau-\Gamma_{i}(s)\right) \mathrm{d} \tau, \\
& \quad i \neq j=1,2, \Gamma_{i}(s) \leq t, \\
& c_{i 0}(t)=c_{i 0}(0) \exp \{-(g+m) t\} \\
& \quad+k \int_{0}^{t} c_{e}(s) \exp \{(s-t)(g+m)) \mathrm{d} s, \\
& c_{e}(t)=c_{e}(0) \exp \left\{-\int_{0}^{t}\left(k_{1} P_{1}(\tau)+k_{1} P_{2}(\tau)+h\right) \mathrm{d} \tau\right\} \\
& \quad+\int_{0}^{t}\left(g_{1} c_{10}(s) P_{1}(s)+g_{1} c_{20}(s) P_{2}(s)+v(s)\right) \\
& \quad \cdot \exp \left\{\int_{t}^{s}\left(k_{1} P_{1}(\tau)+k_{1} P_{2}(\tau)+h\right) \mathrm{d} \tau\right\} \mathrm{d} s
\end{aligned}
$$

where $b_{i}(t)=p_{i}(0, t)$, we obtain

$$
\begin{aligned}
& b_{1}(t)=g_{1}(0) p_{1}(0, t) \\
= & \int_{0}^{\Gamma_{1}^{-1}(t)} \beta_{1}\left(s, c_{10}(t)\right) p_{1}(s, t) \mathrm{d} s \\
\leq & \int_{0}^{\Gamma_{1}^{-1}(t)} \beta_{1}\left(s, c_{10}(t)\right)\left[b_{1}\left(t-\Gamma_{1}(s)\right)\right. \\
& \left.+\int_{0}^{\Gamma_{1}(s)} f_{1}\left(\Gamma_{1}^{-1}(\tau), c_{10}\left(t+\tau-\Gamma_{1}(s)\right)\right) \mathrm{d} \tau\right] \mathrm{d} s \\
\leq & \beta^{0} \int_{0}^{t} b_{1}(\sigma) \mathrm{d} \sigma+\beta^{0}\left\|f_{1}(\cdot, \cdot)\right\|_{L^{1}(Q)} .
\end{aligned}
$$

By Bellman's lemma, we get that

$$
b_{1}(t) \leq \beta^{0}\left\|f_{1}(\cdot, \cdot)\right\|_{L^{1}(Q)} \exp \left(\beta^{0} T\right) .
$$

We define a mapping:

$$
\begin{gathered}
F: X \rightarrow X, \\
F\left(p, c_{0}, c_{e}\right)=\left(F_{1}\left(p, c_{0}, c_{e}\right), \ldots, F_{5}\left(p, c_{0}, c_{e}\right)\right),
\end{gathered}
$$

where

$$
\begin{gathered}
F_{1}\left(p, c_{0}, c_{e}\right)=p_{1}(s, t), F_{2}\left(p, c_{0}, c_{e}\right)=p_{2}(s, t), \\
F_{3}\left(p, c_{0}, c_{e}\right)=c_{10}(t), F_{4}\left(p, c_{0}, c_{e}\right)=c_{20}(t), \\
F_{5}\left(p, c_{0}, c_{e}\right)=c_{e}(t),
\end{gathered}
$$

and $F\left(p, c_{0}, c_{e}\right) \in X$. We can see that

$$
\begin{aligned}
& \int_{0}^{l}\left|F_{1}\left(p, c_{0}, c_{e}\right)\right|(s, t) \mathrm{d} s \\
\leq & \int_{0}^{\Gamma_{1}^{-1}(t)}\left[b_{1}\left(t-\Gamma_{1}(s)\right)\right. \\
& \left.+\int_{0}^{\Gamma_{1}(s)} f_{1}\left(\Gamma_{1}^{-1}(\tau), c_{10}\left(t+\tau-\Gamma_{1}(s)\right)\right) \mathrm{d} \tau\right] \mathrm{d} s \\
\leq & \int_{0}^{t} b_{1}(\sigma) \mathrm{d} \sigma+\left\|f_{1}(\cdot, \cdot)\right\|_{L^{\prime}(Q)} \\
\leq & \beta^{0} T\left\|f_{1}(\cdot, \cdot)\right\|_{L^{\prime}(Q)} \exp \left(\beta^{0} T\right)+\left\|f_{1}(\cdot, \cdot)\right\|_{L^{1}(Q)}:=M_{1} .
\end{aligned}
$$

Similarly, we have

$$
\begin{aligned}
& \int_{0}^{l}\left|F_{2}\left(p, c_{0}, c_{e}\right)\right|(s, t) \mathrm{d} s \\
& \leq \beta^{0} T \exp \left\{2 \lambda^{0} l M_{1}+\beta^{0} \exp \left(\lambda^{0} l M_{1}\right) T\right\}\left\|f_{2}(\cdot, \cdot)\right\|_{L^{1}(Q)} \\
& \quad+\exp \left\{\lambda^{0} l M_{1}\right\}\left\|f_{2}(\cdot, \cdot)\right\|_{L^{1}(Q)}:=M_{2} .
\end{aligned}
$$

Next, we will prove that $F$ is a contracting operator. Let $x^{1}\left(p^{1}, c_{0}^{1}, c_{e}^{1}\right)$ and $x^{2}\left(p^{2}, c_{0}^{2}, c_{e}^{2}\right)$ be the solutions of system (1), then we have

$$
\begin{aligned}
& \int_{0}^{l}\left|F_{1}\left(x^{1}\right)-F_{1}\left(x^{2}\right)\right| \mathrm{d} s \\
& \leq \int_{0}^{\Gamma_{1}^{-1}(t)} \int_{0}^{l} \mid \beta_{1}\left(r, c_{10}^{1}\left(t-\Gamma_{1}(r)\right)\right) p_{1}^{1}\left(r, t-\Gamma_{1}(r)\right) \\
& \quad-\beta_{1}\left(r, c_{10}^{2}\left(t-\Gamma_{1}(r)\right)\right) p_{1}^{2}\left(r, t-\Gamma_{1}(r)\right) \mid \mathrm{d} r \mathrm{~d} s \\
& \quad+\int_{0}^{\Gamma_{1}^{-1}(t)} \beta^{0} M_{1} \int_{0}^{\Gamma_{1}(s)} \mid \mu_{1}\left(\Gamma_{1}^{-1}(\tau), c_{10}^{1}\left(t+\tau-\Gamma_{1}(s)\right)\right) \\
& \quad-\mu_{1}\left(\Gamma_{1}^{-1}(\tau), c_{10}^{2}\left(t+\tau-\Gamma_{1}(s)\right)\right) \mid \mathrm{d} \tau \mathrm{d} s \\
& \quad+\int_{0}^{\Gamma_{1}^{-1}(t)} \beta^{0} M_{1} \int_{0}^{\Gamma_{1}(s)} \lambda^{0}\left(P_{2}^{1}\left(t+\tau-\Gamma_{1}(r)\right)\right. \\
& \left.\quad-P_{2}^{2}\left(t+\tau-\Gamma_{1}(r)\right)\right) \mathrm{d} \tau \mathrm{d} s \\
& +B \int_{0}^{\Gamma_{1}^{-1}(t)} \int_{0}^{\Gamma_{1}(s)} \int_{\tau}^{\Gamma_{1}(s)}\left(\mu_{1}\left(\Gamma_{1}^{-1}(\sigma), c_{10}^{1}\left(t+\sigma-\Gamma_{1}(s)\right)\right)\right. \\
& \left.-\mu_{1}\left(\Gamma_{1}^{-1}(\sigma), c_{10}^{2}\left(t+\sigma-\Gamma_{1}(s)\right)\right)\right) \mathrm{d} \sigma \mathrm{d} \tau \mathrm{d} s
\end{aligned}
$$




$$
\begin{aligned}
& +B \int_{0}^{\Gamma_{1}^{-1}(t)} \int_{0}^{\Gamma_{1}(s)} \int_{\tau}^{\Gamma_{1}(s)} \lambda^{0}\left(P_{2}^{1}\left(t+\sigma-\Gamma_{1}(s)\right)\right. \\
& \left.-P_{2}^{2}\left(t+\sigma-\Gamma_{1}(s)\right)\right) \mathrm{d} \sigma \mathrm{d} \tau \mathrm{d} s \\
& \left.\leq \beta^{0} \int_{0}^{\Gamma_{1}^{-1}(t)} \int_{0}^{l} \mid p_{1}^{1}\left(r, t-\Gamma_{1}(r)\right)-p_{1}^{2}\left(r, t-\Gamma_{1}(r)\right)\right) \mathrm{d} r \mathrm{~d} s \\
& +\left(\beta^{0} M_{1} L_{\mu} l+B l^{2} L_{\mu}+M_{1} L_{\beta}\right) \int_{0}^{\Gamma_{1}^{-1}(t)} \mid c_{10}^{1}\left(t+\tau-\Gamma_{1}(s)\right) \\
& -c_{10}^{2}\left(t+\tau-\Gamma_{1}(s)\right) \mid \mathrm{d} s+\left(\beta^{0} \lambda^{0} l M_{1}+B \lambda^{0} l^{2}\right) \\
& \cdot \int_{0}^{\Gamma_{1}^{-1}(t)} \int_{0}^{\Gamma_{1}(s)}\left(p_{2}^{1}\left(\sigma, t-\Gamma_{1}(r)\right)-p_{2}^{2}\left(\sigma, t-\Gamma_{1}(r)\right)\right) \mathrm{d} \tau \mathrm{d} s \\
& \leq M_{3}\left(\int_{0}^{t} \int_{0}^{l}\left|p_{1}^{1}(r, s)-p_{1}^{2}(r, s)\right| \mathrm{d} s \mathrm{~d} \sigma\right. \\
& \quad+\int_{0}^{t} \int_{0}^{l}\left|p_{2}^{1}(r, s)-p_{2}^{2}(r, s)\right| \mathrm{d} r \mathrm{~d} s \\
& \left.\quad+\int_{0}^{t}\left|c_{10}^{1}(s)-c_{10}^{2}(s)\right| \mathrm{d} s\right),
\end{aligned}
$$

where $M_{3}=\max \left\{\beta^{0}, \beta^{0} \lambda^{0} l M_{1}+B \lambda^{0} l^{2}\right.$,

$$
\left.\beta^{0} M_{1} L_{\mu} l+B l^{2} L_{\mu}+M_{1} L_{\beta}\right\} .
$$

Similarly, we have

$$
\begin{aligned}
& \left|F_{2}\left(x^{1}\right)-F_{2}\left(x^{2}\right)\right| \\
\leq & M_{4}\left(\int_{0}^{t} \int_{0}^{l}\left|p_{2}^{1}(r, s)-p_{2}^{2}(r, s)\right| \mathrm{d} s \mathrm{~d} \sigma\right. \\
& \left.+\int_{0}^{t}\left|c_{20}^{1}(s)-c_{20}^{2}(s)\right| \mathrm{d} s\right),
\end{aligned}
$$

where $M_{4}=\max \exp \left(\lambda^{0} l M_{1}\right)\left\{\beta^{0}, \beta^{0} M_{1} L_{\mu} l+B l^{2} L_{\mu}+M_{2} L_{\beta}\right\}$.

$$
\begin{aligned}
& \left|F_{3}\left(x^{1}\right)-F_{3}\left(x^{2}\right)\right| \\
= & \mid k \int_{0}^{t} c_{e}^{1}(s) \exp \{(s-t)(g+m)\} \mathrm{d} s \\
& -k \int_{0}^{t} c_{e}^{2}(s) \exp \{(s-t)(g+m)\} \mathrm{d} s \mid \\
& \leq M_{5} \int_{0}^{t}\left|c_{e}^{1}(s)-c_{e}^{1}(s)\right| \mathrm{d} s,
\end{aligned}
$$

Similarly,

$$
\begin{aligned}
& \left|F_{4}\left(x^{1}\right)-F_{4}\left(x^{2}\right)\right| \\
\leq & M_{6} \int_{0}^{t}\left|c_{e}^{1}(s)-c_{e}^{1}(s)\right| \mathrm{d} s,
\end{aligned}
$$

where $M_{5}=M_{6}=k$.

$$
\begin{aligned}
& \left|F_{5}\left(x^{1}\right)-F_{5}\left(x^{2}\right)\right| \\
= & \mid c_{e}(0) \exp \left\{-\int_{0}^{t}\left(k_{1} P_{1}^{1}(\tau)+k_{1} P_{2}^{1}(\tau)+h\right)\right\} \\
& +\int_{0}^{t}\left(g_{1} c_{10}^{1}(s) P_{1}^{1}(s)+g_{1} c_{20}^{1}(s) P_{2}^{1}(s)+v(s)\right) \\
& \cdot \exp \left\{\int_{t}^{s}\left(k_{1} P_{1}^{1}(\tau)+k_{1} P_{2}^{1}(\tau)+h\right) \mathrm{d} \tau\right\} \mathrm{d} s \\
& -c_{e}(0) \exp \left\{-\int_{0}^{t}\left(k_{1} P_{1}^{2}(\tau)+k_{1} P_{2}^{2}(\tau)+h\right) \mathrm{d} \tau\right\} \\
& -\int_{0}^{t}\left(g_{1} c_{10}^{2}(s) P_{1}^{2}(s)+g_{1} c_{20}^{2}(s) P_{2}^{2}(s)+v(s)\right) \\
& \cdot \exp \left\{\int_{t}^{s}\left(k_{1} P_{1}^{1}(\tau)+k_{1} P_{2}^{1}(\tau)+h\right) \mathrm{d} \tau\right\} \mathrm{d} s \mid
\end{aligned}
$$

$$
\begin{aligned}
\leq & \int_{0}^{t} \mid g_{1}\left(c_{10}^{1}(s) P_{1}^{1}(s)+c_{20}^{1}(s) P_{2}^{1}(s)\right) \\
& \cdot \exp \left\{\int_{t}^{s} k_{1}\left(P_{1}^{1}(\tau)+P_{2}^{1}(\tau)\right) \mathrm{d} \tau\right\} \\
& -g_{1}\left(c_{10}^{2}(s) P_{1}^{2}(s)+c_{20}^{2}(s) P_{2}^{2}(s)\right) \\
& \cdot \exp \left\{\int_{t}^{s} k_{1}\left(P_{1}^{2}(\tau)+P_{2}^{2}(\tau)\right) \mathrm{d} \tau\right\} \mid \mathrm{d} s \\
& +k_{1} \int_{0}^{t}\left(\left|P_{1}^{1}(\tau)-P_{1}^{2}(\tau)\right|+\left|P_{2}^{1}(\tau)-P_{2}^{2}(\tau)\right|\right) \mathrm{d} \tau \\
& +\int_{0}^{t} \mid v(s)\left(\exp \left\{\int_{t}^{s} k_{1}\left(P_{1}^{1}(\tau)+P_{2}^{1}(\tau)\right) \mathrm{d} \tau\right\}\right. \\
& \left.-\exp \left\{\int_{t}^{s} k_{1}\left(P_{1}^{1}(\tau)+P_{2}^{1}(\tau)\right) \mathrm{d} \tau\right\}\right) \mid \mathrm{d} s \\
\leq & \left(k_{1}+g_{1}\right) \int_{0}^{t}\left(\left|P_{1}^{1}(s)-P_{1}^{2}(s)\right|+\left|P_{2}^{1}(s)-P_{2}^{2}(s)\right|\right) \mathrm{d} s \\
& +g_{1} M \int_{0}^{t}\left(\left|c_{10}^{1}(s)-c_{10}^{2}(s)\right|+\left|c_{20}^{1}(s)-c_{20}^{2}(s)\right|\right) \mathrm{d} s \\
& +\left(2 g_{1} k_{1} M+k_{1} v_{1}\right) \int_{0}^{t} \int_{0}^{\tau}\left(\left|P_{1}^{1}(s)-P_{1}^{2}(s)\right|+\left|P_{2}^{1}(s)-P_{2}^{2}(s)\right|\right) \mathrm{d} \mathrm{d} \tau \\
\leq & M_{7}\left(\sum_{i=1}^{2} \int_{0}^{t} \int_{0}^{l}\left|p_{i}^{1}(r, s)-p_{i}^{2}(r, s)\right| \mathrm{d} r \mathrm{~d} s\right. \\
& \left.+\sum_{i=1}^{2} \int_{0}^{t}\left|c_{i 0}^{1}(s)-c_{10}^{2}\right| \mathrm{d} s\right)
\end{aligned}
$$

where $\quad M_{7}=\max \left\{k_{1}+g_{1}+k_{1} v_{1} T+2 k_{1} g_{1} M T, g_{1} M\right\}$ ， $M=\max \left\{M_{1}, M_{2}\right\}$.

We define an equivalent norm in space $X$ by $\left\|\left(p, c_{0}, c_{e}\right)\right\|_{*}=\operatorname{Ess} \sup _{t \in[0, T]} e^{-\lambda t}\left\{\sum_{i=1}^{2} \int_{0}^{l} p_{i}(s, t) \mathrm{d} t+\sum_{i=1}^{2} c_{i 0}(t)|+| c_{e}(t) \mid\right\}$, where $\lambda>0$ large enough. By (6)-(10), we get $\left\|F\left(x^{1}\right)-F\left(x^{2}\right)\right\|_{*}$ $=\left\|\left(F_{1}\left(x^{1}\right)-F_{1}\left(x^{2}\right), F_{2}\left(x^{1}\right)-F_{2}\left(x^{2}\right), \ldots, F_{5}\left(x^{1}\right)-F_{5}\left(x^{2}\right)\right)\right\|_{*}$ $\leq M_{8}$ Ess $\sup _{t \in[0, T]} e^{-\lambda t} \int_{0}^{t}\left\{\sum_{i=1}^{2} \int_{0}^{l}\left|p_{i}^{1}(r, s)-p_{i}^{2}(r, s)\right| \mathrm{d} r\right.$ $\left.+\sum_{i=1}^{2}\left|c_{i 0}^{1}(s)-c_{i 0}^{2}(s)\right|+\left|c_{e}^{1}(s)-c_{e}^{2}(s)\right|\right\} \mathrm{d} s$ $=M_{8}$ Ess $\sup _{t \in[0, T]} e^{-\lambda t} \int_{0}^{t} e^{\lambda s}\left\{e^{-\lambda s}\left[\sum_{i=1}^{2} \int_{0}^{l}\left|p_{i}^{1}(r, s)-p_{i}^{2}(r, s)\right| \mathrm{d} r\right.\right.$ $\left.+\sum_{i=1}^{2}\left|c_{i 0}^{1}(s)-c_{i 0}^{2}(s)\right|+\left|c_{e}^{1}(s)-c_{e}^{2}(s)\right|\right\} \mathrm{d} s$ $\leq M_{8}\left\|x^{1}-x^{2}\right\|_{*}$ Ess $\sup _{t \in[0, T]}\left\{e^{-\lambda t} \int_{0}^{t} e^{\lambda s} \mathrm{~d} s\right\}$ $\leq \frac{M_{8}}{\lambda}\left\|x^{1}-x^{2}\right\|_{*}$,

where $M_{8}=\max \left\{M_{3}, M_{4}, M_{5}, M_{6}, M_{7}\right\}$. It obvious that for any $\lambda>M_{8}$, then $F$ is a contraction on the space of $\left(X,\|\cdot\|_{*}\right)$ and owns a unique fixed point. Namely, $\left(p, c_{0}, c_{e}\right)$ is the solution of the state system (1).

Lemma 3.1.3 For any $\left(u^{i}, v^{i}\right) \in \Omega$, the state system (1) 
exists a unique non-negative and bounded solution, where $\left|p_{i}(s, t)\right| \leq C_{1}$. Moreover, there exists $K_{i}>0$, such that

$$
\begin{aligned}
& \sum_{i=1}^{2}\left\|p_{i}^{1}-p_{i}^{2}\right\|_{L^{\infty}\left(0, T ; L^{1}(0, l)\right)}+\sum_{i=1}^{2}\left\|c_{i 0}^{1}-c_{i 0}^{2}\right\|_{L^{\infty}(0, T)}+\left\|c_{e}^{1}-c_{e}^{2}\right\|_{L^{\infty}(0, T)} \\
& \leq K_{1} T\left(\sum_{i=1}^{2}\left\|u_{i}^{1}-u_{i}^{2}\right\|_{L^{\infty}\left(0, T ; L^{1}(0, l)\right)}+\left\|v^{1}-v^{2}\right\|_{L^{\infty}(0, T)}\right), \\
& \quad \sum_{i=1}^{2}\left\|p_{i}^{1}-p_{i}^{2}\right\|_{L^{1}(Q)}+\sum_{i=1}^{2}\left\|c_{i 0}^{1}-c_{i 0}^{2}\right\|_{L^{1}(0, T)}+\left\|c_{e}^{1}-c_{e}^{2}\right\|_{L^{1}(0, T)} \\
& \leq K_{2} T\left(\sum_{i=1}^{2}\left\|u_{i}^{1}-u_{i}^{2}\right\|_{L^{1}(Q)}+\left\|v^{1}-v^{2}\right\|_{L^{1}(0, T)}\right) .
\end{aligned}
$$

The proof process of lemma 3.1.3 is similar to that of Theorem 3.1.2, which is omitted here.

\subsection{Optimality conditions}

In this section, we consider the adjoint system of (1) and establish first-order necessary conditions for optimal harvesting of (2).

Lemma 3.2.1 Let $\left(p^{*}, c_{0}^{*}, c_{e}^{*}\right)$ be the solution of system (1) corresponding to $\left(u^{*}, v^{*}\right) \in \Omega$. For any $\left(v_{1}, v_{2}\right) \in T_{\Omega}\left(u^{*}, v^{*}\right), u^{*}=\left(u_{1}^{*}, u_{2}^{*}\right), v_{1}=\left(v_{11}, v_{21}\right)$, such that $\left(u^{*}+\varepsilon v_{1},+v^{*}+\varepsilon v_{2}\right) \in \Omega$ for sufficiently small $\varepsilon>0$, we obtain

$$
\begin{gathered}
\frac{1}{\varepsilon}\left(p_{i}^{\varepsilon}(s, t)-p_{i}^{*}(s, t)\right) \rightarrow z_{i}(s, t), \\
\frac{1}{\varepsilon}\left(c_{i 0}^{\varepsilon}(t)-c_{i 0}^{*}(t)\right) \rightarrow z_{i+2}(t), \\
\frac{1}{\varepsilon}\left(c_{e}^{\varepsilon}(t)-c_{e}^{*}(t)\right) \rightarrow z_{5}(t),
\end{gathered}
$$

as $\varepsilon \rightarrow 0$, where $\left(p^{\varepsilon}, c_{0}^{\varepsilon}, c_{e}^{\varepsilon}\right)$ is the solution of (1) corresponding to $\left(u^{*}+\varepsilon v_{1}, v^{*}+\varepsilon v_{2}\right) \in \Omega \quad$ and $\left(z_{1}, z_{2}, z_{3}, z_{4}, z_{5}\right)$ is the solution of the following system

$$
\begin{aligned}
& \int \frac{\partial z_{1}}{\partial t}+g_{1} \frac{\partial z_{1}}{\partial s}=-\left[\mu_{1}\left(s, c_{10}^{*}(t)\right)+\lambda_{1} P_{2}^{*}(t)+g_{1 s}(s)\right. \\
& \left.+u_{1}^{*}(s, t)\right] z_{1}(s, t)-\int_{0}^{l} \lambda_{1} p_{1}^{*}(s, t) z_{2}(s, t) \mathrm{d} s \\
& -\frac{\partial \mu_{1}\left(s, c_{10}^{*}(t)\right)}{\partial c_{10}} p_{1}^{*}(s, t) z_{3}(t)-v_{11} p_{1}^{*}(s, t), \\
& \frac{\partial z_{2}}{\partial t}+g_{2} \frac{\partial z_{2}}{\partial s}=-\left[\mu_{2}\left(s, c_{20}^{*}(t)\right)-\lambda_{2} P_{1}^{*}(t)+g_{2 s}(s)\right. \\
& \left.+u_{2}^{*}(s, t)\right] z_{2}(s, t)+\int_{0}^{l} \lambda_{2} p_{2}^{*}(s, t) z_{1}(s, t) \mathrm{d} s \\
& -\frac{\partial \mu_{2}\left(s, c_{20}^{*}(t)\right)}{\partial c_{20}} p_{1}^{*}(s, t) z_{4}(t)-v_{21} p_{2}^{*}(s, t), \\
& \frac{\mathrm{d} z_{3}}{\mathrm{~d} t}=k z_{5}(t)-g z_{3}(t)-m z_{3}(t), \\
& \frac{\mathrm{d} z_{4}}{\mathrm{~d} t}=k z_{5}(t)-g z_{4}(t)-m z_{4}(t), \\
& \frac{\mathrm{d} z_{5}}{\mathrm{~d} t}=-k_{1} c_{e}^{*}(t)\left(Z_{1}(t)+Z_{2}(t)\right)+g_{1} c_{10}^{*}(t) Z_{1}(t) \\
& +g_{2} c_{20}^{*}(t) Z_{2}(t)+g_{1}\left(P_{1}^{*}(t) z_{3}(t)+P_{2}^{*}(t) z_{4}(t)\right) \\
& -\left[k_{1}\left(P_{1}^{*}(t)+P_{2}^{*}(t)\right)+h\right] z_{5}(t)+v_{2}, \\
& g_{i}(0) z_{i}(0, t)=\int_{0}^{l} \beta_{i}\left(s, c_{i 0}^{*}(t)\right) z_{i}(t) \mathrm{d} s \\
& +\int_{0}^{l} \frac{\partial \beta\left(s, c_{i 0}^{*}(t)\right)}{\partial c_{i 0}} p_{i}^{*}(s, t) z_{i+2}(t) \mathrm{d} s, \\
& z_{i}(s, t)=z_{i}(s, t+T), \\
& z_{i+2}(0)=z_{5}(0)=0 \text {, } \\
& Z_{i}(t)=\int_{0}^{l} z_{i}(s, t) \mathrm{d} s, P_{i}^{*}(t)=\int_{0}^{l} p_{i}^{*}(s, t) \mathrm{d} s .
\end{aligned}
$$

Theorem 3.2.2 Let $\left(u^{*}, v^{*}\right)$ be an optimal policy for the optimal harvesting problem of (1)-(2). Then

$$
\begin{gathered}
u_{i}^{*}(s, t)=L_{i}\left(\frac{\left[w_{i}(s, t)-q_{i}(s, t)\right] p_{i}^{*}}{c_{i}}\right), i=1,2, \\
v^{*}(t)=L_{3}\left(\frac{q_{5}(t)}{c_{3}}\right),
\end{gathered}
$$

in which $L_{i}$ are given by

$$
L_{j}(\eta)(s, t)= \begin{cases}0, & \eta(s, t)<0, \\ \eta(s, t), & 0 \leq \eta(s, t) \leq H_{j}, j=1,2,3 \\ H_{j}, & \eta(s, t)>H_{j},\end{cases}
$$

where $\left(q_{1}, q_{2}, q_{3}, q_{4}, q_{5}\right)$ is the solution of the following adjoint system (12): 


$$
\begin{aligned}
& \int \frac{\partial q_{1}}{\partial t}+g_{1} \frac{\partial q_{1}}{\partial s}=\left[\mu_{1}\left(s, c_{10}^{*}(t)\right)+\lambda_{1}(s, t) P_{2}^{*}(t)\right. \\
& \left.+u_{1}^{*}(s, t)\right] q_{1}(s, t)+\left(k_{1} c_{e}^{*}(t)-g_{1} c_{0}^{*}(t)\right) q_{5}(t) \\
& -q_{1}(0, t) \beta_{1}\left(s, c_{10}^{*}(t)\right)+w_{1}(s, t) u_{1}^{*}(s, t), \\
& \frac{\partial q_{2}}{\partial t}+g_{2} \frac{\partial q_{2}}{\partial s}=\left[\mu_{2}\left(s, c_{20}^{*}(t)\right)-\lambda_{2}(s, t) P_{1}^{*}(t)\right. \\
& \left.+u_{2}^{*}(s, t)\right] q_{2}(s, t)+\left(k_{1} c_{e}^{*}(t)-g_{1} c_{20}^{*}(t)\right) q_{5}(t) \\
& -q_{2}(0, t) \beta_{2}\left(s, c_{20}^{*}(t)\right)+w_{2}(s, t) u_{2}^{*}(s, t), \\
& \frac{\mathrm{d} q_{3}(t)}{\mathrm{d} t}=\int_{0}^{l} \frac{\partial \mu_{1}\left(s, c_{10}^{*}(t)\right)}{\partial c_{10}} p_{1}^{*}(s, t) q_{1}(x, t) \mathrm{d} s \\
& +(g+m) q_{3}(t)-g_{1} P_{1}^{*}(t) q_{5}(t) \\
& -q_{1}(0, t) \int_{0}^{l} \frac{\partial \beta_{1}\left(s, c_{10}^{*}(t)\right)}{\partial c_{10}} p_{1}^{*}(s, t) \mathrm{d} s, \\
& \frac{\mathrm{d} q_{4}(t)}{\mathrm{d} t}=\int_{0}^{l} \frac{\partial \mu_{2}\left(s, c_{20}^{*}(t)\right)}{\partial c_{10}} p_{2}^{*}(s, t) q_{2}(x, t) \mathrm{d} s \\
& +(g+m) q_{4}(t)-g_{1} P_{2}^{*}(t) q_{5}(t) \\
& -q_{2}(0, t) \int_{0}^{l} \frac{\partial \beta_{2}\left(s, c_{20}^{*}(t)\right)}{\partial c_{20}} p_{2}^{*}(s, t) \mathrm{d} s, \\
& \frac{\mathrm{d} q_{5}(t)}{\mathrm{d} t}=-k q_{3}(t)-k q_{4}(t) \\
& +\left(k_{1} P_{1}^{*}(t)+k_{1} P_{2}^{*}(t)+h\right) q_{5}(t), \\
& q_{i}(l, t)=0 \text {, } \\
& q_{i}(s, t)=q_{i}(s, t+T), \\
& q_{i+1}(T)=q_{i+2}(T)=0, i=1,2 \text {. }
\end{aligned}
$$

Proof For any given element of the tangent cone $\left(v_{1}, v_{2}\right) \in T_{\Omega}\left(u^{*}, v^{*}\right)$, we have $\left(u^{*}+\varepsilon v_{1}, v^{*}+\varepsilon v_{2}\right) \in \Omega$ for any sufficiently small $\varepsilon>0$. Then, due to the optimality of $\left(u^{*}, v^{*}\right)$, it is derived that

$$
\begin{aligned}
& \sum_{i=1}^{2} \int_{0}^{T} \int_{0}^{l} w_{i}(s, t)\left(u_{i}^{*}+\varepsilon v_{i 1}\right) p_{i}^{\varepsilon}(s, t) \mathrm{d} s \mathrm{~d} t \\
- & \frac{1}{2} \sum_{i=1}^{2} \int_{0}^{T} \int_{0}^{l} c_{i}\left(u_{i}^{*}+\varepsilon v_{i 1}\right) \mathrm{d} s \mathrm{~d} t-\frac{1}{2} \int_{0}^{T} c_{3}\left(v^{*}+\varepsilon v_{2}\right) \mathrm{d} t \\
\leq & \sum_{i=1}^{2} \int_{0}^{T} \int_{0}^{l} w_{i}(s, t) u_{i}^{*}(s, t) p_{i}^{*}(s, t) \mathrm{d} s \mathrm{~d} t \\
- & \frac{1}{2} \sum_{i=1}^{2} \int_{0}^{T} \int_{0}^{l} c_{i}\left[u_{i}^{*}(s, t)\right]^{2} \mathrm{~d} s \mathrm{~d} t-\frac{1}{2} \int_{0}^{T} c_{3}\left[v^{*}(t)\right]^{2} \mathrm{~d} t .
\end{aligned}
$$

By Lemma 3.2.1, it follows that

$$
\begin{gathered}
\sum_{i=1}^{2} \int_{0}^{T} \int_{0}^{l} w_{i}(s, t) u_{i}^{*}(s, t) z_{i}(s, t) \mathrm{d} s \mathrm{~d} t \\
+\sum_{i=1}^{2} \int_{0}^{T} \int_{0}^{l}\left[w_{i}(s, t) p_{i}^{*}(s, t)-c_{i} u_{i}^{*}(s, t)\right] v_{i 1}(s, t) \mathrm{d} s \mathrm{~d} t
\end{gathered}
$$

$$
-\int_{0}^{T} c_{3} v^{*}(t) v_{2}(t) \mathrm{d} t \leq 0
$$

Multiplying the first five equations in (11) by $q_{1}(s, t), q_{2}(t), \ldots, q_{5}(t)$ respectively, then integrating on $Q$ and $(0, T)$, combining (13), we get

$$
\begin{aligned}
& \sum_{i=1}^{2} \int_{0}^{T} \int_{0}^{l} w_{i}(s, t) u_{i}^{*}(s, t) z_{i}(s, t) \mathrm{d} s \mathrm{~d} t \\
= & -\sum_{i=1}^{2} \int_{0}^{T} \int_{0}^{l} v_{i 1}(s, t) p_{i}^{*}(s, t) q_{i}(s, t) \mathrm{d} s \mathrm{~d} t \\
& +\int_{0}^{T} v_{2}(t) q_{5}(t) \mathrm{d} t .
\end{aligned}
$$

Substituting (14) into (13) gives that

$$
\begin{aligned}
& \quad \sum_{i=1}^{2} \int_{0}^{T} \int_{0}^{l}\left(\left(w_{i}-q_{i}\right) p_{i}^{*}-c_{i} u_{i}^{*}\right) v_{i 1} \mathrm{~d} s \mathrm{~d} t \\
& +\int_{0}^{T}\left(-c_{3} v^{*}+q_{5}\right) v_{2} \mathrm{~d} t \leq 0,
\end{aligned}
$$

for every $\quad\left(v_{1}, v_{2}\right) \in T_{\Omega}\left(u^{*}, v^{*}\right) \quad$ thus $\left(\left(w_{i}-q_{i}\right) p_{i}^{*}-c_{i} u^{*},-c_{3} v^{*}+q_{5}\right) \in N_{\Omega}\left(u^{*}, v^{*}\right) \quad$, where $N_{\Omega}\left(u^{*}, v^{*}\right)$ is normal cone of $\Omega$ at $\left(u^{*}, v^{*}\right)$.

Lemma 3.2.3 For any $\left(u^{i}, v^{i}\right) \in \Omega, i=1,2$, The adjoint system (12) exists a unique bounded solution, where $\left|q_{i}\right| \leq C_{2}$. Moreover, there exists $K_{3}>0$, such that

$$
\begin{aligned}
& \sum_{i=1}^{2}\left\|q_{i}^{1}-q_{i}^{2}\right\|_{L^{\infty}(Q)}+\sum_{\xi=3}^{4}\left\|q_{\xi}^{1}-c_{\xi}^{2}\right\|_{L^{\infty}(0, T)}+\left\|q_{5}^{1}-q_{5}^{2}\right\|_{L^{\infty}(0, T)} \\
\leq & K_{3} T\left(\sum_{i=1}^{2}\left\|u_{i}^{1}-u_{i}^{2}\right\|_{L^{\infty}(Q)}+\left\|v^{1}-v^{2}\right\|_{L^{\infty}(0, T)}\right) .
\end{aligned}
$$

\subsection{Existence of optimal policy}

In this section, the existence of optimal policy will be established. First, define the embedding mapping $\widetilde{J}(u, v)$ in $\left[L^{1}(Q)\right]^{2} \times L^{1}(0, T)$ as follows

$$
\tilde{J}(u, v)= \begin{cases}J(u, v), & (u, v) \in \Omega, \\ -\infty, & (u, v) \notin \Omega .\end{cases}
$$

Lemma 3.3.1 The functional $\widetilde{J}(u, v)$ is upper semicontinuous in $\left[L^{1}(Q)\right]^{2} \times L^{1}(0, T)$.

Proof Suppose $\left(u^{n}, v^{n}\right) \rightarrow(u, v)$ as $n \rightarrow \infty,\left(p^{n}, c_{0}^{n}, c_{0}^{n}\right)$ and $\left(p, c_{0}, c_{e}\right)$ be solutions of (1) corresponding to $\left(u^{n}, v^{n}\right)$ and $(u, v)$, respectively. According to the Riesz theorem, there exists a subsequence (still denoted by $\left.\left(u^{n}, v^{n}\right)\right)$, it follows that

$$
\begin{gathered}
{\left[u^{n}(s, t)\right]^{2} \rightarrow u^{2}(s, t), \text { a.e. }(s, t) \in Q, \text { as } n \rightarrow \infty .} \\
{\left[v^{n}(t)\right]^{2} \rightarrow v^{2}(t), \text { a.e. } t \in(0, T) \text {, as } n \rightarrow \infty .}
\end{gathered}
$$

From (15) and (16), using Lebesgue dominated convergence theorem implies that 


$$
\begin{gathered}
\lim _{n \rightarrow \infty} \int_{0}^{T} \int_{0}^{l}\left[u_{i}^{n}(s, t)\right]^{2} \mathrm{~d} s \mathrm{~d} t=\int_{0}^{T} \int_{0}^{l}\left[u_{i}(s, t)\right]^{2} \mathrm{~d} s \mathrm{~d} t, \\
\lim _{n \rightarrow \infty} \int_{0}^{T}\left[v^{n}(t)\right]^{2} \mathrm{~d} t=\int_{0}^{T} v^{2}(t) \mathrm{d} t
\end{gathered}
$$

By Lemma 3.1.3, we have

$$
\begin{aligned}
& \quad \mid \int_{0}^{T} \int_{0}^{l} w_{i}(s, t) u_{i}^{n}(s, t) p_{i}^{n}(s, t) \mathrm{d} s \mathrm{~d} t \\
& \quad-\int_{0}^{T} \int_{0}^{l} w_{i}(s, t) u_{i}(s, t) p_{i}(s, t) \mathrm{d} s \mathrm{~d} t \mid \\
& \leq \int_{0}^{T} \int_{0}^{l} w_{i}(s, t) p_{i}^{n}(s, t)\left|u_{i}^{n}(s, t)-u(s, t)_{i}\right| \mathrm{d} s \mathrm{~d} t \\
& \quad+\int_{0}^{T} \int_{0}^{l} w_{i}(s, t) u_{i}(s, t) \mid p_{i}^{n}(s, t)-p_{i}(s, t) \mathrm{d} s \mathrm{~d} t \\
& \leq M \bar{w}\left\|u_{i}^{n}-u_{i}\right\|_{L^{\prime}(Q)}+\bar{w} H\left\|p^{n}-p\right\|_{L^{\prime}(Q)} \\
& \leq M \bar{w}\left\|u_{i}^{n}-u_{i}\right\|_{L^{l}(Q)}+\bar{w} H K_{2} T\left(\left\|u_{i}^{n}-u_{i}\right\|_{L^{\prime}(Q)}+\left\|v^{n}-v\right\|_{L^{\prime}(0, T)}\right), \\
& \text { we get that } \\
& \qquad \lim _{n \rightarrow \infty} \int_{0}^{T} \int_{0}^{l} w_{i}(s, t) u_{i}^{n}(s, t) p_{i}^{n}(s, t) \mathrm{d} s \mathrm{~d} t \\
& \quad=\int_{0}^{T} \int_{0}^{l} w_{i}(s, t) u_{i}(s, t) p_{i}(s, t) \mathrm{d} s \mathrm{~d} t .
\end{aligned}
$$

Therefore, we have proved that

$$
\lim _{n \rightarrow \infty} \sup _{\left(u^{n}, v^{n}\right) \in \Omega} \widetilde{J}\left(u^{n}, v^{n}\right) \leq \widetilde{J}(u, v) .
$$

Theorem 3.3.2 If $\left[c_{1}^{-1} T\left(\bar{w} K_{1}+C_{2} K_{1}+C_{1} K_{3}\right)+c_{2}^{-1} K_{3} T\right] \leq 1$, the optimal harvesting problem (1) and (2) exist a unique optimal policy.

Proof According to Lemma 3.3.1 and Ekeland's variational principle, for each $\varepsilon>0$, there is a $\left(u^{\varepsilon}, v^{\varepsilon}\right) \in \Omega$ such that

$$
\begin{aligned}
\widetilde{J}\left(u^{\varepsilon}, v^{\varepsilon}\right) & \geq \sup _{(u, v) \in \Omega} \widetilde{J}(u, v)-\varepsilon \\
\widetilde{J}\left(u^{\varepsilon}, v^{\varepsilon}\right) & \geq \sup _{(u, v) \in \Omega}\{\widetilde{J}(u, v) \\
& \left.-\sqrt{\varepsilon}\left(\sum_{i=1}^{2}\left\|u_{i}^{\varepsilon}-u_{i}\right\|_{L^{\prime}(Q)}+\left\|v^{\varepsilon}-v\right\|_{L^{\prime}(0, T)}\right)\right\} .
\end{aligned}
$$

By Theorem 3.2.2, we have

$$
\begin{gathered}
\left(w_{i}-q_{i}^{\varepsilon}\right) p_{i}^{\varepsilon}-c_{i} u_{i}^{\varepsilon}+\sqrt{\varepsilon} \theta_{i}^{\varepsilon} \in N_{\Omega}\left(u_{i}^{\varepsilon}\right), i=1,2, \\
q_{5}^{\varepsilon}-c_{3} v^{\varepsilon}+\sqrt{\varepsilon} \theta_{3}^{\varepsilon} \in N_{\Omega}\left(v^{\varepsilon}\right) .
\end{gathered}
$$

Consequently

$$
\begin{gathered}
\left(u^{\varepsilon}, v^{\varepsilon}\right)=\left(L_{1}\left(\frac{\left(w_{1}-q_{1}^{\varepsilon}\right) p_{1}^{\varepsilon}}{c_{1}}+\frac{\sqrt{\varepsilon} \theta_{1}^{\varepsilon}}{c_{1}}\right),\right. \\
\left.L_{2}\left(\frac{\left(w_{2}-q_{2}^{\varepsilon}\right) p_{2}^{\varepsilon}}{c_{2}}+\frac{\sqrt{\varepsilon} \theta_{2}^{\varepsilon}}{c_{2}}\right), L_{3}\left(\frac{q_{5}^{\varepsilon}}{c_{3}}+\frac{\sqrt{\varepsilon} \theta_{3}^{\varepsilon}}{c_{3}}\right)\right),
\end{gathered}
$$

where $\theta_{1}^{\varepsilon}, \theta_{2}^{\varepsilon} \in L^{\infty}(Q), \theta_{3}^{\varepsilon} \in L^{\infty}(0, T)$ with $\left|\theta_{1}^{\varepsilon}\right| \leq 1$, $\left|\theta_{2}^{\varepsilon}\right| \leq 1,\left|\theta_{3}^{\varepsilon}\right| \leq 1$,

First, we prove the uniqueness of optimal harvesting by the contraction mapping principle.

$$
F(u, v)=\left(L_{1}\left(\frac{\left(w_{1}-q_{1}\right) p_{1}}{c_{1}}\right), L_{2}\left(\frac{\left(w_{2}-q_{2}\right) p_{2}}{c_{2}}\right), L_{3}\left(\frac{q_{5}}{c_{3}}\right)\right) .
$$

Therefore, we have

$$
\begin{aligned}
& \left|F\left(u^{1}, v^{1}\right)-F\left(u^{2}, v^{2}\right)\right| \\
\leq & c_{1}^{-1}\left|\left(w_{1}-q_{1}^{1}\right) p_{1}^{1}-\left(w_{2}-q_{1}^{2}\right) p_{1}^{2}\right| \\
+ & c_{2}^{-1}\left|\left(w_{2}-q_{2}^{1}\right) p_{2}^{1}-\left(w_{2}-q_{2}^{2}\right) p_{2}^{2}\right|+c_{3}^{-1}\left|q_{5}^{1}-q_{5}^{2}\right| .
\end{aligned}
$$

Combining Lemma 3.1.3 with Lemma 3.2.3, we get that

$$
\left\|F\left(u^{1}, v^{1}\right)-F\left(u^{2}, v^{2}\right)\right\|
$$

$\leq\left[T\left(c_{1}^{-1}+c_{2}^{-1}\right)\left(\bar{w} K_{1}+C_{2} K_{1}+C_{1} K_{3}\right)+c_{3}^{-1} K_{3} T\right]$

$$
\cdot\left(\sum_{i=1}^{2}\left\|u_{i}^{1}-u_{i}^{2}\right\|_{L^{\infty}(Q)}+\left\|v^{1}-v^{2}\right\|_{L^{\infty}(0, T)}\right) \text {. }
$$

If $\left[T\left(c_{1}^{-1}+c_{2}^{-1}\right)\left(\bar{w} K_{1}+C_{2} K_{1}+C_{1} K_{3}\right)+c_{3}^{-1} K_{3} T\right]<1$, the mapping $F$ is a contraction and has a unique fixed point $(\bar{u}, \bar{v}) \in \Omega$. Then the Theorem 3.2.2 implies that any optimal control $\left(u^{*}, v^{*}\right)$, if exists must be a fixed point of the mapping $F$.

Next we will show that $(\bar{u}, \bar{v}) \in \Omega$ is the optimal policy. Since

$$
\begin{aligned}
& \left\|F\left(u^{\varepsilon}, v^{\varepsilon}\right)-\left(u^{\varepsilon}, v^{\varepsilon}\right)\right\| \\
= & \|\left(L_{1}\left(\frac{\left(w_{1}-q_{1}^{\varepsilon}\right) p_{1}^{\varepsilon}}{c_{1}}\right), L_{2}\left(\frac{\left(w_{2}-q_{2}^{\varepsilon}\right) p_{2}^{\varepsilon}}{c_{2}}\right), L_{3}\left(\frac{q_{5}^{\varepsilon}}{c_{3}}\right)\right) \\
- & \left(L_{1}\left(\frac{\left(w_{1}-q_{1}^{\varepsilon}\right) p_{1}^{\varepsilon}}{c_{1}}+\frac{\sqrt{\varepsilon} \theta_{1}}{c_{1}}\right), L_{2}\left(\frac{\left(w_{2}-q_{2}^{\varepsilon}\right) p_{2}^{\varepsilon}}{c_{2}}+\frac{\sqrt{\varepsilon} \theta_{2}}{c_{2}}\right),\right. \\
& \left.L_{3}\left(\frac{q_{5}^{\varepsilon}}{c_{3}}+\frac{\sqrt{\varepsilon} \theta_{3}}{c_{3}}\right)\right) \| \\
\leq & c_{1}^{-1} \sqrt{\varepsilon}\left\|\theta_{1}^{\varepsilon}\right\|+c_{2}^{-1} \sqrt{\varepsilon}\left\|\theta_{2}^{\varepsilon}\right\|+c_{3}^{-1} \sqrt{\varepsilon}\left\|\theta_{3}^{\varepsilon}\right\| \\
\leq & \sqrt{\varepsilon}\left(c_{1}^{-1}+c_{2}^{-1}+c_{3}^{-1}\right) .
\end{aligned}
$$

and $F(\bar{u}, \bar{v})=(\bar{u}, \bar{v})$, it follows that

$\left\|(\bar{u}, \bar{v})-\left(u^{\varepsilon}, v^{\varepsilon}\right)\right\|$

$\leq\left\|F(\bar{u}, \bar{v})-F\left(u^{\varepsilon}, v^{\varepsilon}\right)\right\|+\left\|F\left(u^{\varepsilon}, v^{\varepsilon}\right)-\left(u^{\varepsilon}, v^{\varepsilon}\right)\right\|$

$\leq\left[T\left(c_{1}^{-1}+c_{2}^{-1}\right)\left(\bar{w} K_{1}+C_{2} K_{1}+C_{1} K_{3}\right)+c_{3}^{-1} K_{3} T\right]$

$\cdot\left(\sum_{i=1}^{2}\left\|\bar{u}_{i}-u_{i}^{\varepsilon}\right\|_{L^{\infty}(Q)}+\left\|\bar{v}-v^{\varepsilon}\right\|_{L^{\infty}(0, T)}\right)+\sqrt{\varepsilon}\left(c_{1}^{-1}+c_{2}^{-1}+c_{3}^{-1}\right)$

$\leq\left(\max \left\{K_{3}, K_{4}\right\}\right) T\left(c_{1}^{-1}+c_{2}^{-1}+c_{3}^{-1}\right)$

$\cdot\left(\sum_{i=1}^{2}\left\|\bar{u}_{i}-u_{i}^{\varepsilon}\right\|_{L^{\infty}(Q)}+\left\|\bar{v}-v^{\varepsilon}\right\|_{L^{\infty}(0, T)}\right)+\sqrt{\varepsilon}\left(c_{1}^{-1}+c_{2}^{-1}+c_{3}^{-1}\right)$,

that is

$$
\begin{aligned}
& \sum_{i=1}^{2}\left\|\bar{u}_{i}-u_{i}^{\varepsilon}\right\|_{L^{\infty}(Q)}+\left\|\bar{v}-v^{\varepsilon}\right\|_{L^{\infty}(0, T)} \\
& \leq \frac{\sqrt{\varepsilon}\left(c_{1}^{-1}+c_{2}^{-1}+c_{3}^{-1}\right)}{\left(1-\max \left\{K_{3}, K_{4}\right\}\right) T\left(c_{1}^{-1}+c_{2}^{-1}+c_{3}^{-1}\right)} .
\end{aligned}
$$

where $\bar{w} K_{1}+C_{2} K_{1}+C_{1} K_{3}=K_{4}$.

Therefore $\left(u^{\varepsilon}, v^{\varepsilon}\right) \rightarrow(\bar{u}, \bar{v})$ as $\varepsilon \rightarrow 0$. From Lemma 
3.3.1 and inequality (17) it follows that

$$
\widetilde{J}(\bar{u}, \bar{v})=\sup _{(u, v) \in \Omega} \widetilde{J}(u, v),
$$

which implies that $(\bar{u}, \bar{v}) \in \Omega$ is the optimal policy.

\section{Conclusion}

In this paper, an optimal development model of a periodic population system dependent on sized-structure in a polluted environment is established and analyzed. If all the parameters in the optimal control problem (1) and (2) are periodic functions related to time, then with the increase of time, the total population resources will show periodic changes. From the Theorem 3.2.2 and 3.3.2, the optimal harvesting problem (1) and (2) have at least one optimal harvesting policy, and the Theorem 3.2.2 shows that the optimal harvesting policy has a Bang-Bang structure. The optimal harvesting policy in Theorem 3.2.2 provides a threshold condition for optimal harvesting problems (1) and (2):

$$
\left(w_{i}-q_{i}\right) p_{i}^{*}-c_{i} u^{*},-c_{3} v^{*}+q_{5}, i=1,2 .
$$

If $\left(w_{i}-q_{i}\right) p_{i}^{*}-c_{i} u^{*}<0, q_{5}-c_{3} v^{*}<0$, that is, when the economic value of the population is relatively low, then the optimal harvesting policy is equal to its minimum value 0 . If $\left(w_{i}-q_{i}\right) p_{i}^{*}-c_{i} u^{*}>0, q_{5}-c_{3} v^{*}>0$, that is, when the economic value of the population is relatively high, then the optimal harvesting policy is equal to its maximum value $H_{j}, j=1,2,3$. This result has obvious practical significance.

In practical applications, the parameter values in models (1) and (2) are fitted by observation data. Then combined with (2), (3) and (12) to calculate the optimal harvest policy and the optimal population density. Furthermore, the optimal index $s$ can be obtained through numerical calculation, which provides a theoretical basis for the development and utilization of population resources.

\section{Acknowledgment}

The authors would like to thank the reviewers for excellent suggestions that improved the presentation of this paper. This worked is supported by the Nature Science Foundation of China (11561041).

\section{References}

[1] T. G. Hallam, C. E. Clark, R. R., Lassiter. Effects of toxicants on population: A qualitative approach I. Equilibrium environmental exposure. Ecol. Model., 18: 291-304 (1983)

[2] T. G. Hallam, C. E. Clark, G. S. Jordan. Effects of toxicants on populations: A qualitative approach II . First order kinetics. J. Math. Biol., 18: 25-37 (1983)

[3] T. G. Hallam, J. T. De Luna. Effects of toxicants on populations: A qualitative approach III. Environmental and food chain pathways. J. theor.
Biol., 109: 411-429 (1984)

[4] Z. X. Luo, Z. R. He. Optimal control of agedependent population hybrid system in a polluted environment. Appl. Math. Comput., 228: 68-76 (2014)

[5] H. Wang, F. M. Pan, M. Liu. Survival analysis of a stochastic service-resource mutualism model in a polluted environment with pulse toxicant input. Physica A, 521: 591-606 (2019)

[6] G. D. Liu, X. Z. Meng. Optimal harvesting strategy for a stochastic mutualism system in a polluted environment with regime switching. Physica $A, \mathbf{5 3 6}$ 120893 (2019)

[7] S. Anita. Analysis and Control of Age-Dependent Population Dynamics. Kluwer Academic Publishers, Dordrecht (2000)

[8] Y. J. Li, Z. H. Zhang, Y. F. Lv, et al. Optimal harvesting for a size-stage-structured population model. Nonlinear Anal. Real World Appl., 44: 616630 (2018)

[9] J. Liu, X. S. Wang. Numerical optimal control of a size-structured PDE model for metastatic cancer treatment. Math. Biosic., 314: 28-42 (2019)

[10] A. Skonhoft, V. Friberg. Optimal harvesting in the presence of predation: An age-structured modelling approach. J. Environ. Manage., 227: 111341 (2021)

[11] Z. R. He, R. Liu. Theory of optimal harvesting for a nonlinear size-structured population in periodic environments. Int. J. Biomath., 7: 1450046 (2014)

[12] F. Q. Zhang, R. Liu, Y. M. Chen. Optimal harvesting in a periodic food chain model with size structures in predators. Appl. Math. Optim., 75: 229-251 (2017)

[13] L. I. Anita, S. Anita. Note on some periodic optimal harvesting problems for age-structured population dynamics. Appl. Math. Comput., 276: 21-30 (2016)

[14] Y. Liu, X. L. Cheng, Z. R. He. On the optimal harvesting of size-structured population dynamics. Appl. Math. J. Chinese Univ., 28: 173-186 (2013) 